Revista de

Contabilidade e

Organizações

www.rco.usp.br
DOI: http://dx.doi.org/10.11606/issn.1982-6486.rco.2018.143279
Journal of

Accounting and

Organizations

www.rco.usp.br

\title{
Por que as empresas realizam aquisições com frequência?
}

\section{Why do companies frequently acquire?}

Daiana Paula Pimenta; ${ }^{\mathrm{a}}$ Rafael Barreiros Porto ${ }^{\mathrm{b}}$

${ }^{a}$ Universidade Federal de Goiás

${ }^{b}$ Universidade de Brasilia

Palavras-chave

Adquirentes Frequentes.

Experiência em Aquisições.

Aquisições Corporativas.
Keywords

Frequent Acquirers.

Acquisition Experience.

Corporate Acquisition.
Informações do Artigo

Recebido: 07 de fevereiro de 2018

Aceito: 21 de agosto de 2018

Publicado: 27 de agosto de 2018

\section{Resumo}

Apesar da relevância das aquisições frequentes como um programa estratégico corporativo, sabe-se pouco sobre os motivos de sua ocorrência. Os estudos focam na identificação dos fatores que determinam a primeira aquisição, desconsiderando que as empresas podem se envolver em sucessivos eventos. Para preencher essa lacuna, este estudo identifica os motivos que levam as empresas a se tornarem adquirentes frequentes, comparando com o que já se sabe sobre as motivações para realização da primeira aquisição. Por meio de Regressões Logísticas e de Poisson, identificamos que os preditores da primeira aquisição contribuem para explicar a frequência desses eventos. Ainda, a frequência de aquisições pode ser vista como uma estratégia para manter a competitividade da empresa, cujo principal propulsor é o auto interesse dos principais executivos.

\begin{abstract}
Despite the relevance of frequent acquisitions as a corporate strategic program, little is known about the reasons for its occurrence. The studies focus on identifying the factors that determine the first acquisition, disregarding that companies can engage in successive events. To fill this gap, this study identifies the reasons that lead companies to become frequent acquirers, comparing with what is already known about the motivations for the first acquisition. Through Logistic and Poisson Regressions, we identified that the predictors of the first acquisition contribute to explain the frequency of occurrence of these events. As well as, the frequency of acquisitions could be considered as a strategy to support the business competitiveness, which its main driver is the executives' self-interest.
\end{abstract}

\section{Implicações práticas}

Empresas realizam aquisições com frequência como parte de uma estratégia, segundo características do setor e associado à proporção de ações em posse do principal executivo e sua presença no conselho. A frequência está menos associada às condições financeiras da empresa adquirente no ano da operação. Estes resultados mostram que para o mercado norte-americano empresas empregam aquisições como padrão estratégico, e não como ações isoladas.

Copyright (C) 2018 FEA-RP/USP. Todos os direitos reservados

\section{INTRODUÇÃO}

As aquisições são uma estratégia crucial no mundo corporativo. Entre os anos de 2012 e 2017 mais de 27 trilhões de dólares foram investidos nestes eventos em todo o mundo (Statista, 2018). Em paralelo a esta importância prática, tanto em termos financeiros quanto estratégicos, as atividades de aquisição tornaram-se foco de estudo em diversos campos acadêmicos, tais como gestão estratégica, finanças e sociologia.

Autor Correspondente: Tel. (62) 3521-1000

E-mail:daiana-pimenta@hotmail.com (D. P. Pimenta); rafaelporto@unb.br (R. B. Porto)

Universidade Federal de Goiás. Avenida Esperança s/n - Chácaras de Recreio Samambaia, Goiânia - GO, 74690-900, Brasil 
As pesquisas sobre esta temática geraram um considerável conhecimento e revelaram aspectos importantes sobre as tendências e características deste fenômeno (Arikan \& Stulz, 2016; Dodd, 1980; Ghosh, 2004; Haleblian, Devers, McNamara, Carpenter, \& Davison , 2009). Uma das principais linhas de investigação fundamentou-se na identificação dos antecedentes das aquisições (Dietrich \& Sorensen, 1984; Erdogan, 2012; Hannan \& Pilloff, 2009; Palepu, 1986). Uma gama de fatores determinantes relacionados ao auto interesse gerencial, aos fatores ambientais e às características da empresa e do negócio foram identificados e extensamente pesquisados (ver Haleblian et al., 2009).

Todavia, ao analisar os antecedentes das aquisições, as pesquisas, em sua maioria, consideram apenas aquisições focais, normalmente, a primeira aquisição realizada por uma empresa (Dietrich \& Sorensen, 1984; Erdogan, 2012; Hannan \& Pilloff, 2009; Palepu, 1986). Dessa forma, desconsideram que as empresas podem se envolver em sucessivas aquisições durante sua existência. Fato que é intrigante, pois basta uma análise geral dos dados sobre aquisições para se perceber que realizar aquisições frequentemente é um comportamento corporativo cada vez mais comum (Al Rahahleh \& Wei, 2012).

A frequência de aquisições tem sido reconhecida por alguns estudiosos como um mecanismo a partir do qual a empresa aprende, por meio da experiência, a gerir melhor os processos de aquisição (Aktas, de Bodt, \& Roll, 2013; Haleblian et al., 2009). Por outro lado, há pesquisadores que ainda são céticos a esse respeito (Hayward, 2002; Kusewitt, 1985; Zollo \& Singh, 2004). Isso porque, se o argumento central é aprender a adquirir por meio da experiência, esperar-se-ia que quanto mais a empresa faz aquisições, melhor o desempenho das aquisições e, consequentemente, maior a frequência com que realiza esse tipo de negócio.

No entanto, o aumento da frequência de aquisições não parece ser motivado pelo desempenho obtido pela empresa em eventos anteriores, pois essas continuam a fazer aquisições mesmo quando as pesquisas relatam relações negativas ou nulas entre a experiência e o desempenho de uma aquisição focal (Hayward, 2002; Kusewitt, 1985; Zollo \& Singh, 2004).

A simples acumulação de experiência não influencia o desempenho da empresa. Apenas nos casos em que as empresas adquirentes conseguem discriminar adequadamente entre suas aquisições, ou seja, separar as aquisições que são similares em termos setoriais daquelas que não são, é que a codificação do conhecimento pode influenciar positivamente o desempenho da aquisição (Haleblian \& Finkelstein, 1999; Zollo \& Sing, 2004). Ou seja, é preciso tempo para aprender a adquirir e, nesse intervalo, é fato que a frequência aumenta, pois $60 \%$ de todas as aquisições realizadas no mundo são feitas por adquirentes frequentes (Henningsson, 2014).

Pelo exposto, as organizações podem ignorar os resultados obtidos em aquisições anteriores. Assim, é possível que o comportamento da firma, no que se refere a realizar aquisições frequentemente, dependa da experiência, mas não do desempenho dos eventos passados. Isso porque, as empresas tendem a ignorar as inferências em termos de desempenho das aquisições anteriores, principalmente, quando essas geram dúvidas sobre os méritos de uma aquisição focal (Haunschild, Davis-Blake, \& Fichman, 1994). Fato que é reforçado em função de as aquisições frequentes serem consideradas um programa estratégico, que já em uma fase inicial tem o seu impacto no desempenho de longo prazo da firma planejado (Barkema \& Schijven, 2008).

A frequência de aquisições parece ser influenciada pela experiência da empresa com esse tipo de evento. Tal fato demonstra que as variáveis clássicas, consideradas determinantes da primeira aquisição, são insuficientes para explicar esse comportamento. Além disso, as pesquisas têm feito inferência sobre os determinantes da frequência de aquisições de forma fragmenta, deixando uma visão unificada sobre as motivações subjacentes a este comportamento como uma notável lacuna desta literatura.

Neste contexto, esta pesquisa analisa os motivos que levam as empresas a se tornarem adquirentes frequentes, a partir do que já se sabe sobre as motivações das primeiras aquisições. Utilizamos os clássicos determinantes das aquisições em conjunto com a experiência nessas atividades para explicar o comportamento de aquisição frequente pelas firmas. Foram realizadas regressões Logísticas e de Poisson, com dados longitudinais, para analisar uma amostra de 1.286 empresas estadunidenses de capital aberto. A pesquisa é percursora ao apresentar evidências empíricas de que os preditores clássicos da primeira aquisição contribuem, em conjunto com a experiência da empresa com essas atividades, para explicar o comportamento de aquisição. Além disso, nossos resultados são uma importante contribuição para explicar parte dos motivos que levam as organizações a realizarem aquisições frequentemente, mesmo quando há consenso de que os resultados obtidos com essas atividades, na maioria das vezes, são nulos ou negativos. 


\section{REFERENCIAL TEÓRICO E HIPÓTESES DA PESQUISA}

\subsection{Determinantes das aquisições}

A literatura apresentou uma gama de motivos que explicam a ocorrência da primeira aquisição de uma empresa. Dentre estes, está o auto interesse gerencial, representado pela ganância e busca de engrandecimento pessoal de executivos (Ferreira et al., 2014; Haleblian et al., 2009). O auto interesse gerencial, constitui-se em uma das principais explicações para a destruição de valor em aquisições (Capron \& Pistre, 2002). Para Brown e Sarma (2007) os retornos negativos para os acionistas das empresas adquirentes podem ser explicados pelos custos de agência, ou seja, pelo fato de os executivos realizarem aquisições para aumentar sua riqueza e status. Da mesma forma, pode ser explicado pelo poder do principal executivo da empresa, uma vez que este é o membro mais poderoso da elite corporativa e pode buscar impor sua vontade sobre os outros membros (Brown \& Sarma, 2007).

A literatura aponta também diferentes características da empresa, do negócio e do ambiente como motores das aquisições. As características da empresa representam os traços que podem contribuir ou dificultar a participação de uma empresa em atividades de aquisição (Haleblian et al., 2009). Dentre estas, destacam-se o tamanho da empresa, o fluxo de caixa livre, a alavancagem e o desempenho da empresa (Haleblian et al., 2009; Masulis, Wang \& Xie, 2007).

No que se refere ao tamanho, Akhigbe, Madura e Whyte (2004) demonstram que a probabilidade de as maiores empresas adquirirem as menores é maior em função dos altos custos de transação e de integração pós-negócio. No caso das adquirentes frequentes, em particular, o tamanho é um preditor em função de poder influenciar as escolhas estratégicas de uma organização, pois grandes empresas estão sujeitas a forças de inércia e, portanto, são mais propensas a repetir suas ações anteriores (Haleblian, Kim, \& Rajagoplan, 2006). O fluxo de caixa livre e a alavancagem são fatores antecedentes, pois as empresas com mais recursos disponíveis e/ou com uma melhor capacidade de obtenção de financiamento externo são mais propensas a fazer aquisições do que aquelas com menor capacidade (Masulis et al., 2007).

O desempenho da empresa também tem uma atenção especial na literatura de aquisições. Embora esta literatura ainda não aborde os reais efeitos da estratégia de aquisição em série sobre o desempenho de novas aquisições, já foram documentados efeitos do desempenho da empresa adquirente sobre o seu desempenho posterior. Heron e Lie (2002), por exemplo, demonstram que as empresas adquirentes com forte desempenho operacional antes da aquisição, continuaram a ter tal desempenho.

O $Q$ de Tobin, que representa as oportunidades de investimento da empresa, é uma forma de captar esse desempenho, dada sua associação implícita positiva com os fluxos de caixa futuros da empresa (Fu, Singhal \& Parkas, 2016). Outra métrica de desempenho encontrada na literatura de aquisições é o Market Share. Ghosh (2004) afirma que as aquisições são uma forma de a empresa aumentar seu Market Share, buscando maior poder de mercado, reduzindo a concorrência setorial e se beneficiando de rendas de monopolísticas. Nesse sentido, o desempenho da empresa adquirente, possivelmente, influencia a ocorrência desses eventos.

As características do negócio são entendidas como fatores preditores e indicadores de sucesso ou falha das aquisições (Haleblian et al., 2009). Uma das principais características, apontada pela literatura, é o tamanho relativo da aquisição. Os resultados encontrados evidenciam que o valor do negócio influencia positivamente a probabilidade de uma futura aquisição. O que, possivelmente, está relacionado ao fato de que uma maior aquisição pode ser percebida como um evento mais importante do que uma aquisição menor, e caso o desempenho obtido seja positivo, pode aumentar a confiança do adquirente mais do que um resultado semelhante obtido a partir de uma menor aquisição e consequentemente promover ainda mais aquisições (Haleblian, Kim, \& Rajagopalan, 2006).

As características ambientais funcionam como um mecanismo de seleção no sentido evolutivo clássico, atribuindo contexto às aquisições e podendo proporcionar feedback sobre o valor e a viabilidade dos comportamentos atuais da organização (Zollo \& Winter, 2002). A principal característica ambiental, diretamente identificada à probabilidade de ocorrência desses eventos, é a densidade de aquisições no setor. Uma vez que, as empresas tendem a fazer mais aquisições como forma de imitar as estratégias de outras, desejando conseguir os mesmos resultados (Haleblian, Kim \& Rajagoplan, 2006). Pelo exposto, a literatura sobre os determinantes das aquisições é extensa e permite inferir que:

$\mathbf{H}_{1 \mathbf{a}}: \mathrm{O}$ auto interesse gerencial, os fatores ambientais, as características da empresa e do próprio negócio são determinantes da primeira aquisição de uma empresa.

$\mathbf{H}_{1 \mathrm{~b}}$ : $\mathrm{O}$ auto interesse gerencial, os fatores ambientais, as características da empresa e do próprio negócio são determinantes da frequência de aquisições de uma empresa. 


\subsection{Efeitos da experiência sobre a frequência de aquisições}

No tópico anterior argumentamos que o auto interesse gerencial, os fatores ambientais, as características da empresa e do próprio negócio são determinantes da frequência de aquisições de uma empresa. Todavia, ao se envolver em mais de um evento, a empresa começa a adquirir experiência no processo de aquisição, o que, possivelmente, é um fator preditivo para a realização de novos eventos (Haleblian, Kim, \& Rajagoplan, 2006; Peng \& Fang, 2010). Nesse sentido, propomos que a experiência somada aos preditores já conhecidos da primeira aquisição são capazes de explicar a ocorrência frequente desses eventos.

Esse argumento está baseado na literatura sobre aprendizagem comportamental, a qual sugere que o comportamento das firmas é guiado por rotinas sustentadas pela experiência (Levitt \& March, 1988; Nelson \& Winter, 1982). Especificamente, essa literatura defende que as organizações aprendem ao realizar tarefas, sendo o conhecimento adquirido a partir da experiência armazenado em suas rotinas, constituindo-se assim em registros de sua história (Nelson \& Winter, 1982).

Segundo Baum, Li e Usher (2000) as escolhas e ações codificadas em rotinas são mais propensas a ser aceitas pelos membros e tomadores de decisão organizacionais. A literatura oferece evidências de que os membros, detentores de experiência com uma ação estratégica em particular, tendem a repetir a mesma ação posteriormente (Haleblian, Kim \& Rajagopalan, 2006).

Amburgey e Miner (1992) ao explicarem a ocorrência de momentos repetitivos nas atividades de aquisição, chamam essa tendência da organização em manter a direção estratégica de ações anteriores em um comportamento estratégico atual de momento estratégico. As autoras utilizam o conceito de inércia para justificar o fato de as empresas experientes em um tipo de aquisição em particular - vertical, horizontal ou conglomerado estarem mais propensas a seguir o mesmo padrão posteriormente. Haleblian, Kim e Rajagoplan (2006), também apresentam resultados consistentes com a ideia de que as rotinas organizacionais, uma vez estabelecidas, estão sujeitas a pressões inerciais. Os autores demonstram que as empresas adquirentes são mais propensas a fazer aquisições à medida que ganham experiência com um tipo de aquisição em particular, similar ou não.

$\mathrm{Na}$ literatura, as aquisições relacionadas ao mesmo setor de atividade da empresa adquirente, estão, comumente, associadas à busca por maior participação e poder de mercado (Anand \& Singh, 1997; Ghosh, 2004). Já as não relacionadas ao setor de atividade das empresas adquirentes representam aquisições com foco em sinergias financeiras.

Pelo exposto, evidências teóricas e empíricas da literatura postulam uma relação determinista entre a experiência e as ações internas da organização, consistente com as alegações que as empresas são mais propensas a repetir uma ação estratégica quando possuem experiência prévia em tal ação (Gulati, 1995). Isso porque, embora inicialmente os tomadores de decisão das organizações não tenham nenhuma certeza quanto aos resultados de suas atividades, a repetição da ação leva a maior experiência, aumentado a confiança e reduzindo as incertezas à medida que a compreensão e a capacidade de execução das rotinas melhoram (Levitt \& March, 1988). Os argumentos apresentados permitem inferir que:

$\mathbf{H}_{2}$ : A experiência de uma empresa em aquisições anteriores feitas por ela, seja do seu setor de atividade ou de um setor diferente, aumenta sua frequência de aquisições.

\section{METODOLOGIA}

As hipóteses da pesquisa foram testadas por meio de dados longitudinais em uma amostra de empresas estadunidenses não financeiras de capital aberto. As análises englobam o período entre janeiro de 1996 e dezembro de 2014. Os dados foram obtidos em duas bases de dados diferentes e posteriormente combinados. As informações sobre as aquisições foram coletadas na Thomson Financials SDC Database e as financeiras e de mercado na Compustat.

Para formar a amostra de empresas adquirentes, foram selecionados todos os negócios codificados como aquisição de participação majoritária anunciados e concluídos no período entre $1^{\circ}$ de janeiro de 1996 e 31 de dezembro de 2014. Foram mantidas na amostra apenas empresas em que o adquirente controlava menos de 50\% das ações da firma-alvo antes da data do anúncio e mais de $90 \%$ após a aquisição (Bena \& Li, 2014). Assim como, seguindo a literatura sobre o tema, exigiu-se que: (1) o valor do negócio fosse igual ou superior a US\$ 1 milhão (dólares constantes de 1996), a fim de eliminar as transações economicamente insignificantes; (2) a transação fosse incondicional e completa; (3) as empresas adquirentes não fizessem parte do setor financeiro e nem do setor de utilidades e; (4) as adquirentes tivessem, pelo menos, três anos de dados financeiros anteriores às datas de anúncio disponíveis na base de dados (Bena \& Li, 2014). 
Para formar a amostra de controle, levou-se em consideração uma das principais críticas aos estudos sobre aquisição: o fato de muitas vezes as empresas adquirentes serem comparadas a outras que não captam as perspectivas da firma caso não houvesse o negócio, levando a estimativas tendenciosas (Bena \& Li, 2014). Visando minimizar esse problema de heterogeneidade da amostra, para formar o grupo de controle, utilizou-se o pareamento por escore de propensão (propensity score matching), um método que visa reduzir o viés de seleção pela busca de empresas similares ou pareadas em termos de características observáveis (Rosenbaum \& Rubin, 1983).

Para tanto, baixou-se da base de dados Compustat todas as empresas ativas entre 1996 e 2014, excluindo as que fizessem parte do setor financeiro e de utilidades. Posteriormente, excluiu-se desse grupo de empresas aquelas já identificadas como adquirentes na Thomson Financial's SDC Database. A partir dessas informações, iniciou-se o processo de pareamento por meio do software Stata 12. As empresas adquirentes foram combinadas com as não adquirentes primeiro por setor (Standard Industry Classification de 2 dígitos) e, em seguida, pelos escores de propensão estimados a partir do tamanho e dos índices book-to-market.

Para cada aquisição anunciada no ano t foram identificadas no mínimo duas (2) e no máximo três (3) potenciais empresas adquirentes (correspondentes) na base de dados Compustat que não tinham realizado nenhuma aquisição. Como muitas empresas adquirentes foram excluídas da amostra de aquisições em função dos filtros utilizados, estas estavam sujeitas a compor o grupo de controle. Para evitar problemas nas análises, tomou-se o cuidado de verificar se as empresas pareadas do grupo de controle não tinham se envolvido em aquisições durante o período focal observado.

O book-to-market foi incluído nas características de pareamento em função de captar os principais condutores das aquisições: oportunidades de crescimento (Bena \& Li, 2014), sobrevalorização (Shleifer \& Vishny, 2003; Bena \& Li, 2014, Rhodes-Kropf \& Viswanathan, 2004) e complementaridade de recursos (Bena \& Li, 2014; Rhodes-Kropf \& Robinson, 2008).Em função dos procedimentos utilizados, os grupos experimental e de controle tornaram-se comparáveis e quaisquer diferenças observáveis entre esses grupos podem ser consideradas pequenas e devidas ao erro de chance. Tecnicamente, assegurando que a estimativa do resultado contrafactual é imparcial (Roberts \& Whited, 2013), ou seja, que as estimativas dos resultados das empresas adquirentes, caso essas não tivessem se envolvido em aquisições, e dos resultados das empresas da amostra de controle, caso estas tivessem realizado aquisições, não são viesadas.

Após o pareamento, obteve-se uma amostra final com 5.044 empresas, das quais 1.286 realizaram entre 1 e 8 aquisições e 3.758 não se envolveram em nenhuma aquisição, compondo um painel desbalanceado de informações ao longo de 18 anos. Todos os dados de mercado e contábeis foram winsorizados ao nível de 5\%, como uma forma de eliminar os efeitos dos outliers.

\subsection{Variáveis Dependentes}

Primeira aquisição. A utilização de modelos binários para identificação dos determinantes das aquisições é, comumente, encontrada na literatura (Akhigbe et al., 2004; Bena \& Li, 2014, dentre outros). Dessa forma, construímos uma variável dependente qualitativa dicotômica (dummy), em que um valor igual a 1 indica que, naquele ano, a empresa realizou sua primeira aquisição e 0 caso contrário, para testar a hipótese $\mathrm{H}_{1 \mathrm{a}}$ desta pesquisa. Para cada observação de uma empresa que realizou sua primeira aquisição, há várias observações para as empresas de controle. Especificamente, essa variável dependente representa a chance de ocorrência da primeira aquisição para uma determinada empresa i em um instante específico de tempo t.

Frequência de aquisições. A literatura tem considerado a frequência de aquisições como a quantidade de ocorrências de aquisições para determinada empresa i em um específico instante do tempo t. No nosso conhecimento, esta é a primeira pesquisa a utilizar uma variável de frequência, como variável dependente, para verificar o número de ocorrência de aquisições. As demais pesquisas identificadas, utilizaram a frequência como variável explicativa (Hayward, 2004; Haleblian, Kim, \& Rajagopalan, 2006).

Para a construção desta variável, nos fundamentamos nos modelos que podem ser utilizados quando a variável dependente é uma variável de contagem. Portanto, frequência de aquisições é a quantidade anual de aquisições realizada por cada empresa (número de ocorrências). 


\subsection{Variáveis Independentes e de Controle}

Características da empresa. Foram inseridas cinco variáveis para capturar os efeitos das características da empresa. A primeira delas, é o tamanho da empresa, mensurado pelo logaritmo dos ativos totais em dólares (Akhigbe et al., 2004). A segunda, a alavancagem, calculada a partir da divisão das dívidas de longo prazo pelo valor total da firma (Masulis et al., 2007). A terceira é o fluxo de caixa livre, obtido pela dedução dos valores das despesas com juros, imposto de renda e despesas de capital do valor do lucro líquido antes da depreciação (Masulis et al., 2007). A quarta e a quinta variáveis representam o desempenho da firma, cujo $Q$ de Tobin e o Market Share foram utilizados como proxies. O $Q$ de Tobin foi mensurado a partir da divisão do valor de mercado da firma pelo valor de reposição dos ativos (Fu et al., 2016) e, o Market Share, calculado pela divisão do faturamento bruto de uma empresa pelo faturamento bruto de todas as empresas do setor (Ghosh, 2004).

Auto interesse gerencial. O auto interesse gerencial é aproximado por três variáveis. A primeira é o poder do executivo, mensurada a partir da divisão da remuneração do gestor pelos ativos totais da empresa (Brown \& Sarma , 2007). Essa variável representa a capacidade do executivo para impor seus pontos de vista excessivamente otimistas nas decisões da empresa. Um executivo dominante provavelmente exerce o seu domínio na determinação das ações estratégicas da empresa, podendo agir em seu interesse em detrimento do dos acionistas (Brow \& Sarma, 2007, p.363). A segunda, é a proporção de ações em posse do gestor em relação ao volume total de ações da empresa (Cooper, Gulen, \& Rau, 2013). A terceira é a participação do gestor no conselho, em que o valor 1 indica que o gestor é membro do conselho para a empresa i no tempo t e 0 para o caso contrário (Shimizu, 2007).

Características ambientais. As condições ambientais, em particular regulação e incerteza, podem afetar tanto o comportamento de aquisição de uma empresa quanto suas consequências, podendo ser determinantes das escolhas estratégicas corporativas (Haleblian, Kim, \& Rajagopalan, 2006). Visando minimizar potenciais problemas com variáveis omitidas, testamos os efeitos da Sarbanes Oxley em todos os modelos estimados. Essa lei estadunidense, assinada em 30 de julho de 2002, foi motivada por escândalos financeiros corporativos (dentre eles o da Enron, que acabou por afetar drasticamente a empresa de auditoria Arthur Andersen). Em nenhum dos modelos estimados obteve-se efeito ou significância para essa variável. Assim ela não foi incluída nas análises.

Para tomar essa decisão, nos fundamentamos em pesquisas anteriores, as quais identificaram que as atividades de aquisição parecem não ser impedidas por características regulatórias. Matsusaka (1996), por exemplo, ao examinar a influência de ações regulatórias no comportamento de aquisição, descobriu que as leis antitruste não impedem as atividades de aquisição. Rossi e Volpin (2004), corroboram esse achado ao apresentaram evidências de que em países com padrões contábeis e de governança mais elevados, como é o caso dos Estados Unidos, as empresas são mais propensas a realizar aquisições.

Por outro lado, no que se refere à incerteza, Bergh e Lawless (1998) demonstram que em situação de incerteza ambiental decrescente as empresas altamente diversificadas são mais propensas a realizar aquisições do que aquelas menos diversificadas. Já em situações de incerteza crescente, como durante a crise subprime, os mercados financeiros são submetidos a um ambiente volátil e incerto que diminui, significativamente, o número de aquisições no mercado (Gaughan, 2009). A partir desses argumentos, foram inseridas três variáveis para captar os efeitos das características ambientais sobre a probabilidade de uma firma realizar sua primeira aquisição e/ou aumentar a frequência com que se envolve com esses eventos.

A primeira é a densidade de aquisições no setor. Essa variável, mensurada a partir da soma do número de aquisições realizadas por todas as empresas de um determinado setor (Standard Industry Classification de 2 dígitos), foi inserida em função de pesquisas anteriores sugerirem que a intensidade das atividades de aquisição em um setor pode afetar o comportamento de aquisição das empresas individuais (Haleblian, Kim, \& Rajagoplan, 2006).

A segunda variável é a receita do setor, mensurada pela soma das receitas de todas as empresas de um setor a cada ano analisado. Essa variável foi incluída para controlar as mudanças ao longo do tempo e as inerentes às receitas globais da indústria (Haleblian, Kim, \& Rajagopalan, 2006). A terceira variável é a crise subprime, uma dummy em que 0 representa os anos anteriores a 2007, início da crise nos Estados Unidos, e 1 indica o período posterior a 2007.

Características da aquisição. Com base na literatura, foram incluídas três variáveis representativas das características do negócio. A primeira delas é o tamanho relativo da aquisição, mensurado a partir da divisão do valor dos ativos da empresa-alvo pelo valor dos ativos da adquirente (Haleblian, Kim, \& Rajagoplan, 2006). Essa variável foi incluída em função de uma maior aquisição poder ser percebida como um evento mais importante do que uma aquisição menor, podendo aumentar a confiança do adquirente e, consequentemente, promover ainda mais aquisições (Haleblian, Kim, \& Rajagopalan, 2006). 
Amburgey e Miner (1992) argumentam que a similaridade entre a empresa adquirente e a alvo têm influência sobre as futuras aquisições, por isso foram inseridas duas variáveis para capturar esse efeito. A primeira delas, chamada aquisição relacionada, foi obtida pela soma cumulativa do número de empresas de setores semelhantes ao seu (Standard Industry Classification de 2 dígitos) adquiridas por uma licitante ao longo dos anos. A segunda, intitulada de aquisição não relacionada, foi obtida pela soma cumulativa do número de empresas de setores diferentes ao seu (Standard Industry Classification de 2 dígitos) adquiridas por uma empresa ao longo dos anos. Não há relação de complementaridade entre essas variáveis, pois as empresas podem adquirir mais ou menos firmas alvo dentro ou fora de seu setor. Por fim, foram inseridas dummies para controlar os efeitos do tempo e de empresa em todos os modelos estimados.

\subsection{Modelagem Empírica}

A variável dependente utilizada para testar a primeira hipótese, primeira aquisição, é binária. Assim, utilizou-se o modelo de regressão logística binomial com dados em painel. Para verificar a adequabilidade deste modelo, foram aplicados os testes de Hausman-MacFadden (1984), de Small e Hsiao (1985) e alternativamente o Suest-base Hausman, com o objetivo de verificar a independência das alternativas irrelevantes, e, por conseguinte, a suposição de independência entre os temos de erro.

As outras duas hipóteses da pesquisa possuem a frequência de aquisições como variável dependente. Em função de essa variável ser discreta e composta por números inteiros não negativos, a estimação por mínimos quadrados ordinários não forneceria resultados robustos, tornando, portanto, preferível a utilização de modelos adequados para dados de contagem como, por exemplo, a regressão de Poisson (Das \& Kapil, 2015).

O modelo de Poisson tem a forte restrição de que a variância e a média devem ser iguais, suposição que é muitas vezes violada nos conjuntos de dados de contagem real ( $\mathrm{Hu}, 2000)$. Caso os dados apresentem superdispersão, uma alterativa é utilizar o modelo de regressão Binomial Negativo, que corrige o problema adicionando mais variância (heterogeneidade não observada) por meio de um parâmetro $\alpha$ adicional (Colin Cameron \& Trivedi, 1998). Sendo assim, a estratégia empírica deste trabalho consistiu em estimar os modelos de Poisson e Binomial Negativo, utilizando o teste LR para definir o modelo mais adequado. Os resultados do teste não foram significativos (Prob chi2 = 1.0000), demostrando que o modelo de Poisson é o que melhor se adequa. Essa escolha também se respalda nos resultados dos Critérios de informação Bayesiano de Schwarz e Akaike, que foram um pouco melhores para o modelo Poisson.

Para minimizar possíveis problemas de variáveis omitidas, considerando assim a heterogeneidade não observada, optou-se por estimar os modelos da pesquisa com efeitos fixos. Para confirmar a adequabilidade dessa escolha, utilizou-se o teste de Hausman (1978). Além disso, antes de iniciar a estimação dos modelos, foram realizados testes para verificar a sua adequabilidade. Os primeiros testes indicaram a presença de heterocedasticidade e autocorrelação. Como forma de superar esses problemas, aplicaram-se erros padrão robustos agrupados no nível da firma em todas as regressões estimadas. Calculou-se também o fator de inflação da variância das variáveis preditoras e analisou-se as correlações entre elas. Nenhum indício de multicolinearidade foi encontrado.

\section{RESULTADOS}

As estatísticas descritivas para as variáveis utilizadas no estudo estão apresentadas na Tabela 1. Em função do pareamento por score de propensão feito para selecionar a amostra de não adquirentes, percebe-se que as empresas adquirentes e aquelas que compõem a amostra de controle, possuem, em médias, valores de $Q$ de Tobin, receita do setor e tamanho semelhantes. Além disso, o fluxo de caixa livre é, em média, negativo para as empresas que compõem a amostra de controle. Enquanto, para a amostra de adquirentes, o valor médio é positivo. Tal fato se justifica a partir das evidências já apresentadas na literatura de que as empresas com mais fluxo de caixa disponível são mais propensas a realizar aquisições (Harford, 2002). A alavancagem e o Market Share, também não apresentaram diferenças estatisticamente significantes entre a amostra de controle e a de adquirentes.

No que se refere às características gerenciais, a participação no conselho, o poder do executivo e o percentual de ações em posse do gestor são, em média, superiores para as empresas adquirentes. Resultado que vai ao encontro da literatura de aquisições, em relação às influências positivas do auto interesse gerencial sobre a ocorrência de aquisições (Shi et al., 2017). Por fim, é possível observar que as empresas da amostra de adquirente realizaram, em média, mais aquisições de empresas alvo relacionadas ao seu setor de atividade do que empresas não relacionadas. 
Tabela 1. Estatísticas descritivas para as variáveis-chave da pesquisa

\begin{tabular}{|c|c|c|c|c|c|c|c|c|c|c|c|c|c|c|c|}
\hline & \multicolumn{5}{|c|}{ Amostra de Controle } & \multicolumn{5}{|c|}{ Amostra de Adquirentes } & \multicolumn{5}{|c|}{ Amostra Total } \\
\hline Variáveis & $\mathrm{N}$ & Média & $\begin{array}{l}\text { Desvio } \\
\text { Padrão }\end{array}$ & Mín & Máx & $\mathrm{N}$ & Média & $\begin{array}{l}\text { Desvio } \\
\text { Padrão }\end{array}$ & Mín & Máx & $\mathrm{N}$ & Média & $\begin{array}{l}\text { Desvio } \\
\text { Padrão }\end{array}$ & Mín & Máx \\
\hline Frequência de Aquisições & 40075 & 0,00 & 0,00 & 0,00 & 0,00 & 16646 & 0,93 & 0,94 & 1,00 & 8,00 & 56721 & 0,27 & 0,66 & 0,00 & 8,00 \\
\hline Primeira aquisição & 40075 & 0,00 & 0,00 & 0,00 & 0,00 & 16646 & 0,07 & 0,26 & 1,00 & 1,00 & 56721 & 0,02 & 0,15 & 0,00 & 1,00 \\
\hline Tamanho (log ativos totais) & 40075 & 6,55 & 2,03 & 1,93 & 11,82 & 16646 & 6,93 & 1,82 & 1,93 & 11,82 & 56721 & 6,66 & 1,98 & 1,93 & 11,82 \\
\hline Fluxo de caixa livre & 37067 & $-0,04$ & 0,39 & $-0,45$ & 2,8 & 15283 & 0,02 & 0,16 & $-4,65$ & 0,57 & 52350 & $-0,02$ & 0,34 & $-4,50$ & 2,80 \\
\hline Alavancagem & 39924 & 0,16 & 0,17 & 0,00 & 3,21 & 16546 & 0,15 & 0,16 & 0,00 & 0,98 & 56470 & 0,16 & 0,17 & 0,00 & 3,21 \\
\hline$Q$ de Tobin & 40073 & 2,49 & 3,26 & 0,53 & 28,94 & 16605 & 2,36 & 2,49 & 0,54 & 7,15 & 56678 & 2,46 & 3,05 & 0,54 & 28,94 \\
\hline Gestor membro do conselho & 40075 & 0,29 & 0,45 & 0,00 & 1,00 & 16646 & 0,48 & 0,50 & 0,00 & 1,00 & 56721 & 0,34 & 0,47 & 0,00 & 1,00 \\
\hline Poder do executivo & 40075 & 1,22 & 4,75 & 0,00 & 24,88 & 16646 & 1,96 & 6,02 & 0,00 & 22,23 & 56721 & 1,44 & 5,17 & 0,00 & 24,88 \\
\hline Ações em posse do gestor (\%) & 40075 & 0,99 & 4,41 & 0,00 & 63,47 & 16646 & 1,26 & 4,57 & 0,00 & 87,6 & 56721 & 1,07 & 4,47 & 0,00 & 87,6 \\
\hline Densidade de aquisições & 40075 & 0,00 & 0,00 & 0,00 & 0,00 & 16646 & 8,91 & 9,30 & 0,00 & 38 & 56721 & 6,98 & 8,23 & 0,00 & 38 \\
\hline Receita do setor (trilhões USD) & 40075 & 5,56 & 4,43 & 0,72 & 18,5 & 16646 & 5,40 & 3,99 & 1,84 & 18,5 & 56721 & 5,52 & 4,30 & 1,84 & 18,5 \\
\hline Market Share & 40018 & 0,02 & 0,05 & 0,00 & 1,00 & 16609 & 0,02 & 0,05 & 0,00 & 1,00 & 56627 & 0,02 & 0,05 & 0,00 & 1,00 \\
\hline Tamanho relativo da aquisição & 40075 & 0,00 & 0,00 & 0,00 & 0,00 & 16646 & 0,03 & 0,31 & 0,00 & 14,85 & 56721 & 0,03 & 0,31 & 0,00 & 14,85 \\
\hline Crise subprime & 40075 & 0,38 & 0,48 & 0,00 & 1,00 & 16646 & 0,36 & 0,48 & 0,00 & 1,00 & 56721 & 0,37 & 0,48 & 0,00 & 1,00 \\
\hline Aquisições relacionadas & 40075 & 0,00 & 0,00 & 0,00 & 0,00 & 12134 & 0,88 & 0,88 & 0,00 & 8,00 & 12134 & 0,88 & 0,88 & 0,00 & 8,00 \\
\hline Aquisições não relacionadas & 40075 & 0,00 & 0,00 & 0,00 & 0,00 & 5704 & 0,74 & 0,73 & 0,00 & 5,00 & 5704 & 0,74 & 0,73 & 0,00 & 5,00 \\
\hline
\end{tabular}

Fonte: Dados da pesquisa, 2018. 
Na Tabela 2, estão expostas as estimativas da regressão logística, cuja variável dependente é a primeira aquisição. Assim como das regressões de Poisson, em que a variável dependente é a frequência de aquisições.

Tabela 2. Determinantes da primeira aquisição e da frequência de aquisições corporativas

\begin{tabular}{|c|c|c|c|c|c|c|}
\hline Variáveis & \multicolumn{2}{|c|}{ Primeira Aquisição } & \multicolumn{2}{|c|}{ Frequência de Aquisições } & \multicolumn{2}{|c|}{ Frequência de Aquisições } \\
\hline Variáveis preditoras e de controle & $\beta$ & Erro Padrão & $\beta$ & Erro Padrão & $\beta$ & Erro Padrão \\
\hline Tamanho da empresa & $0,318 * * *$ & $(0,101)$ & $0,120 * * *$ & $(0,014)$ & $0,059 * * *$ & $(0,028)$ \\
\hline Fluxo de caixa livre & $-0,024$ & $(0,065)$ & $-0,003$ & $(0,006)$ & 0,017 & $(0,011)$ \\
\hline Alavancagem & $-0,020$ & $(0,073)$ & $-0,018 * *$ & $(0,008)$ & $-0,000^{*}$ & $(0,014)$ \\
\hline$Q$ de Tobin & $0,136^{*}$ & $(0,072)$ & 0,006 & $(0,095)$ & 0,005 & $(0,020)$ \\
\hline Gestor membro do conselho & $0,429 * *$ & $(0,245)$ & $0,048^{*}$ & $(0,026)$ & $0,058^{*}$ & $(0,035)$ \\
\hline Poder do executivo & 0,005 & $(0,086)$ & $0,001^{*}$ & $(0,008)$ & $0,031 * *$ & $(0,013)$ \\
\hline Ações em posse do gestor (\%) & $0,061 * * *$ & $(0,087)$ & $0,000^{*}$ & $(0,010)$ & $0,006^{*}$ & $(0,016)$ \\
\hline Densidade de aquisições & $-0,101$ & $(0,066)$ & $0,011 * *$ & $(0,005)$ & $0,004 * *$ & $(0,024)$ \\
\hline Receira do setor & $-0,174$ & $(0,142)$ & $-0,012$ & $(0,016)$ & $-0,012$ & $(0,016)$ \\
\hline Market Share & $-0,233 * * *$ & $(0,078)$ & $0,020^{*}$ & $(0,011)$ & $0,024 *$ & $(0,022)$ \\
\hline Tamanho relativo da aquisição & $1,474 * * *$ & $(0,044)$ & $0,147 * * *$ & $(0,004)$ & $0,059 * * *$ & $(0,008)$ \\
\hline Crise subprime & $-4,139 * * *$ & $(0,718)$ & $-1,818 * * *$ & $(0,194)$ & $-3,916^{* * *}$ & $(0,739)$ \\
\hline Aquisições relacionadas & & & & & $0,276^{* * *}$ & $(0,043)$ \\
\hline Aquisições não relacionadas & & & & & $0,280 * * *$ & $(0,044)$ \\
\hline Efeitos fixos da empresa & \multicolumn{2}{|c|}{$\operatorname{Sim}$} & \multicolumn{2}{|c|}{$\operatorname{Sim}$} & \multicolumn{2}{|c|}{$\operatorname{Sim}$} \\
\hline Efeitos fixos do tempo & \multicolumn{2}{|c|}{ Sim } & \multicolumn{2}{|c|}{ Sim } & \multicolumn{2}{|c|}{ Sim } \\
\hline Número de observações & \multicolumn{2}{|c|}{14.172} & \multicolumn{2}{|c|}{14.884} & \multicolumn{2}{|c|}{14.884} \\
\hline
\end{tabular}

Fonte: Dados da pesquisa, 2018.

Nota: As variáveis das regressões estão padronizadas. Entre parênteses estão os erros padrão robustos à heterocedasticidade. $*$ p-valor $\leq 0,10 ; * *$ p-valor $\leq 0,05 ; * * *$ p-valor $\leq 0,01$.

O sumário da regressão em que a primeira aquisição é a variável dependente indica que os coeficientes das variáveis tamanho da empresa, percentual de ações do gestor e tamanho relativo da aquisição, foram positivos e significantes ao nível de 1\%. Ao mesmo nível, os coeficientes das variáveis Market Share e crise subprime, apresentaram-se negativos sobre a probabilidade de a empresa realizar sua primeira aquisição.

O $Q$ de Tobin e a participação do gestor no conselho também apresentaram coeficientes positivos e significantes ( $\mathrm{p}$-valor $\leq 0,05$ ). Esses resultados suportam a hipótese $\mathrm{H}_{1 \mathrm{a}}$ desta pesquisa, ao demostrar que, pelo menos, uma variável que representa o auto interesse gerencial, as características da empresa, do ambiente e do negócio configura-se como preditora da primeira aquisição. Para a variável dependente primeira aquisição, os coeficientes das variáveis tamanho da empresa e tamanho relativo da aquisição foram positivos e significantes (p-valor $\leq 0,01)$, e o mesmo ocorre quando a variável dependente é a frequência de aquisições.

Com relação a variável dependente frequência de aquisições, a participação do gestor no conselho, o percentual de ações do gestor, a dominância do gestor, a densidade de aquisições no mercado e o Market Share apresentaram resultados significativamente positivos sobre a probabilidade de a empresa aumentar a frequência de aquisições. Enquanto a alavancagem e a crise subprime, apresentaram efeitos negativos. Esses resultados indicam que, assim como ocorreu com os determinantes da primeira aquisição, pelo menos, uma variável que representa o auto interesse gerencial, as características da empresa, do ambiente e do negócio configurou-se como preditora da frequência de aquisições, suportando assim a hipótese $\mathrm{H}_{1 \mathrm{~b}}$ desta pesquisa.

Por fim, no que se refere aos coeficientes das variáveis que indicam a experiência da empresa com aquisições relacionadas ao setor de atividade da empresa ou não, percebe-se que, ao nível de 1\% de significância, tanto a experiência com a realização de aquisições relacionadas ao setor de atividade da empresa, quanto aquelas não relacionadas, influenciam positivamente o aumento da frequência de aquisições. Esse resultado suporta a hipótese $\mathrm{H}_{2}$ ao demostrar que a experiência da empresa em aquisições se une aos determinantes clássicos para explicar a frequência de ocorrência desses eventos. 


\subsection{Discussão}

Os preditores tradicionais da primeira aquisição, com algumas diferenças, em conjunto com a experiência em aquisições contribuem para explicar a frequência com que as empresas se envolvem com esses eventos. Assim, incorpora à literatura insights úteis sobre os determinantes da estratégia de adquirir em série. Ao observar se os preditores da primeira aquisição também predizem a frequência de aquisições, cinco deles demonstraram efeitos semelhantes. O tamanho da empresa (em ativos totais) tem efeito positivo. Tanto para primeira aquisição, quanto para frequência, empresas de maior porte assimilam os altos custos de transação e de integração pós-negócio (Akhigbe et al., 2004), e pelas forças de inércia são mais propensas a repetir as ações, mantendo a frequência de aquisições (Haleblian, Kim, \& Rajagoplan, 2006).

Um ponto de destaque nos resultados é o papel exercido pelo principal executivo. Todas as três variáveis associadas à presença do executivo estão associadas à frequência de aquisição. A participação do executivo no conselho, o percentual de ações em posse desse executivo e a proporção da remuneração desse executivo em relação aos ativos totais. Parece haver uma forte influência desse executivo, pela presença do mesmo no conselho, e a influência potencial das aquisições no pacote de remuneração do mesmo. Ainda, o tamanho relativo da aquisição influencia tanto a primeira aquisição quanto a continuidade desses eventos. Acreditamos que ao realizarem aquisições, com alto volume de recursos financeiros envolvidos, os executivos buscam alcançar maior domínio do mercado, o que gera maior glamour para empresa e, consequentemente, para o seu cargo (Malmendier \& Tate, 2008; Haleblian, Kim, \& Rajagoplan, 2006).

Em relação à crise subprime, tanto para primeira aquisição quanto para o uso frequente da aquisição, a incerteza gerada no cenário econômico norte-americano apresentou efeito negativo sobre a ocorrência de aquisição. Tal resultado já era esperado, uma vez que todo o sistema financeiro dos Estados Unidos estava sob alto risco e, conforme já apresentado, em períodos de recessão há um declínio nas atividades de aquisição (Martynova \& Renneboog, 2005).

Apenas o Market Share, apesar de significante, apresentou efeitos divergentes. O efeito é negativo para a probabilidade da primeira aquisição, mas positivo sobre a chance de elas aumentarem a frequência com que se envolvem com essas atividades. Este resultado sugere que quando há um aumento no Market Share da empresa a chance de ela realizar sua primeira aquisição é reduzida. Em contrapartida, indica que um aumento no Market Share contribui positivamente para que a empresa aumente a frequência de aquisições. Uma possível explicação para essa situação é que, ao contrário das empresas que realizam sua primeira aquisição, as adquirentes frequentes possuem referências de eventos anteriores. Assim, o aumento no Market Share pode ser associado aos resultados positivos de aquisições realizadas, aumentando a chance de a empresa repetir a mesma ação (Haleblian, Kim, \& Rajagopalan, 2006). Levitt e March (1988) mostram que embora os executivos não tenham nenhuma certeza quanto aos resultados de suas atividades, a repetição da ação leva a maior experiência, aumentado a confiança e a probabilidade de recorrência da ação.

Além disso, as aquisições frequentes são um programa estratégico corporativo, que, já em uma fase inicial, possui o desempenho em longo prazo planejado (Barkema \& Schijven, 2008). Ao analisar as estatísticas descritivas da pesquisa podemos perceber que as empresas adquirentes possuem, em média, a mesma participação de mercado das empresas da amostra de controle. Intuitivamente, acreditamos que um motivo para tal semelhança é a utilização da estratégia de adquirir em série para alcançar maior poder de mercado e tornar-se mais competitiva dentro do setor. Já que existem outras empresas com uma participação de mercado muito semelhante, sem terem se envolvido com nenhum evento.

A variável alavancagem foi significativa apenas nas situações em que a frequência de aquisições é a variável dependente. Os resultados demonstram que um aumento no nível de endividamento, reduz a chance de a empresa aumentar a frequência de aquisições, indicando que a estratégia de adquirir em série se restringe ao volume de endividamento da empresa. O poder do executivo e a densidade de aquisições no mercado também foram significativas apenas nos modelos em que a frequência de aquisições é a variável dependente, ambas com sinais positivos. Tal resultado indica que, o poder do executivo e o fato de outras empresas do mesmo setor estarem envolvidas com aquisições, impactam positivamente a decisão de adquirir em série, mas não a decisão de realizar a primeira aquisição.

Os efeitos do $Q$ de Tobin foram encontrados apenas no que se refere à realização da primeira aquisição. Um aumento no nível de $Q$ de Tobin da empresa exerce efeito positivo sobre a chance de ela realizar sua primeira aquisição, mas não sobre a frequência de aquisições. Este resultado pode ser explicado ao consideramos que o $Q$ de Tobin é uma proxy para as oportunidades de investimento da empresa (Fu et al., 2016) e que, como tal, exerce influência sobre a ocorrência de um investimento feito pela primeira vez. Enquanto que a frequência de aquisições, como um programa estratégico, não depende de um aumento nas oportunidades de investimento da empresa. 
Por fim, a experiência da empresa em aquisições relacionadas ou não ao seu setor de atividade influencia positivamente a ocorrência desses eventos. O número de aquisições relacionadas realizadas pelas adquirentes frequentes é, em média, superior ao número de aquisições não relacionadas. Indicando que ao fazer das aquisições frequentes uma estratégia, as empresas, possivelmente, buscam maior participação e poder de mercado. Além disso, esse resultado corrobora as evidências teóricas e empíricas da literatura de que as empresas são mais propensas a repetir uma ação estratégica quando possuem experiência prévia em tal ação (Gulati, 1995).

\section{CONSIDERAÇÕES FINAIS}

Acreditamos que este estudo seja o primeiro a analisar as diferenças entre a empresa optar por fazer uma primeira aquisição e tornar essa estratégia uma ação frequente. Identificamos que algumas características da empresa, dos executivos, do ambiente e do negócio predeterminam, da mesma forma, tanto a primeira aquisição quanto a ocorrência frequente desses eventos. Estas características estão ligadas ao tamanho da empresa, ao auto interesse dos executivos e aos períodos de recessão no mercado estadunidense.

Todavia, conforme demonstrado, outros fatores exerceram influência sobre a primeira aquisição ou sobre a frequência de aquisições. Dentre aqueles que impactam apenas essa última, estão a alavancagem e a densidade de aquisições. Uma descoberta interessante é que o aumento da alavancagem demostrou um efeito negativo sobre a frequência de ocorrência desses eventos. Outro achado é que as a frequência de aquisições está associada fortemente com um setor de atividade, mas o mesmo não se observa na primeira aquisição. Além disso, quanto maior a participação de mercado, maior é passa a ser a frequência de aquisições, mas o mesmo não ocorre na primeira aquisição. É como se as adquirentes frequentes estivessem sempre em busca de maior poder de mercado, corroborando a hipótese de auto interesse gerencial presente na literatura de aquisições.

Demostramos ainda que a experiência em aquisições da empresa, seja essa relacionada ou não ao setor de atividade da empresa, influencia positivamente o número de ocorrência desses eventos. De uma forma geral, os preditores clássicos da primeira aquisição contribuem, em conjunto com a experiência da empresa com essas atividades, para explicar o comportamento de aquisição frequente das firmas. Essas evidências reunidas sugerem que a frequência de aquisições é uma estratégia, ou um programa corporativo, visando a competitividade da empresa no segmento. Tal estratégia teria como principal propulsor o interesse e atuação dos principais executivos.

Os resultados aqui apresentados são uma primeira análise dos efeitos das variáveis estudadas sobre o comportamento das adquirentes frequentes. Não foram considerados os efeitos da frequência de aquisições sobre o desempenho da firma e de outras proxies que podem representar os antecedentes destes eventos. Além disso, as inferências feitas no que se refere ao Market Share, merecem estudos mais robustos, o que se constitui um fértil campo de estudos.

\section{REFERÊNCIAS}

Akhigbe, A., Madura, J., \& Whyte, A. M. (2004). Partial Anticipation and the Gains to Bank Merger Targets. Journal of Finance Service, 26(1), 55-71. DOI: https://doi.org/10.1023/B:FINA.0000029657.34575.d2

Aktas, N., de Bodt, E., \& Roll, R. (2013). Learning from repetitive acquisitions: Evidence from the time between deals. Journal of Financial Economics, 108(1), 99-117. DOI: https://doi.org/10.1016/j.jfineco.2012.10.010

Al Rahahleh, N., \& Wei, P. (2012). The performance of frequent acquirers: Evidence from emerging markets. Global Finance Journal, 23(1), 16-33. DOI: https://doi.org/10.1016/j.gfj.2012.01.002

Amburgey, T., \& Miner, A. (1992). Strategic momentum: The effects of repetitive, positional, and contextual momentum on merger activity. Strategic Management Journal, 13(5), 335-348. DOI: https://doi.org/10.1002/ smj.4250130503

Anand, J., \& Singh, H. (1997). Asset Re-deployment, Acquisitions and Corporate Strategies in Declining Industries. Strategic Management Journal, 18(Summer Special Issue), 99-118. DOI: https://www.jstor.org/ stable/3088212

Arikan, A., \& Stulz, R. (2016). Corporate acquisitions, diversification, and the firm's life cycle. The Journal of Finance, 71(1), 139-194. DOI: https://doi.org/10.1111/jofi.12362

Barkema, H., \& Schijven, M. (2008). How do firms learn to make acquisitions? a review of past research and an agenda for the future. Journal of Management, 34(3), 594-634. DOI: https://doi.org/10.1177/0149206308316968 
Baum, J. A. C., Li, S., \& Usher, J. (2000). Making the Next Move: How Experiential and Vicarious Learning Shape the Locations of Chains' Acquisitions. Administrative Science Quarterly, 45(4), 766-801. DOI: https://www. jstor.org/stable/2667019

Bena, J., \& Li, K. (2014). Corporate innovations and mergers and acquisitions. The Journal of Finance, 69(5), 1923-1960. DOI: https://doi.org/10.1111/jofi.12059

Bergh, D., \& Lawless, M. (1998). Portfolio Restructuring and Limits to Hierarchical Governance: The Effects of Environmental Uncertainty and Diversification Strategy. Organization Science, 9(1), 87-102. DOI: https:// doi.org/10.1287/orsc.9.1.87

Brown, G., \& Sarma, N. (2007). CEO overconfidence, CEO dominance and corporate acquisitions. Journal of Economics and Business, 59(2), 358-379. DOI: https://doi.org/10.1016/j.jeconbus.2007.04.002

Capron, L., \& Pistre, N. (2002). When do Acquirers Earn Abnormal Returns Strategic Management Journal Strategic Management, 23(1), 781-794. DOI: https://doi.org/10.1002/smj.262

Colin Cameron, A., \& Trivedi, P. K. (1998). Regression analysis of count data. Econometric Society Monographs, 30.

Cooper, M. J., Gulen H., \& Rau, P. R. (2013). Performance for pay? The relation between CEO incentive compensation and future stock price performance, Working paper. The University of Utah. DOI: http://dx.doi. org/10.2139/ssrn.1572085

Das, A., \& Kapil, S. (2015). Inorganic growth of technology sector firms in emerging markets: Influence of firmspecific factors in indian firms m\&a activities. International. Journal of Emerging Markets, 10(1), 52-72. DOI: https://doi.org/10.1108/IJOEM-06-2012-0057

Dietrich, J., \& Sorensen, E. (1984). An application of logit analysis to prediction of merger targets. Journal of Business Research, 12(3), 393-402. DOI: https://doi.org/10.1016/0148-2963(84)90020-1

Dodd, P. (1980). Merger proposals, management discretion and stockholder wealth. Journal of Financial Economics, 8(2), 105-137. DOI: https://doi.org/10.1016/0304-405X(80)90014-8

Erdogan, A. (2012). The determinants of mergers and acquisitions: evidence from turkey. International Journal of Economics and Finance, 4(4), 72. DOI: https://doi.org/10.5539/ijef.v4n4p72

Ferreira, M., Santos, J., de Almeida, M., \& Reis, N. (2014). Mergers \& acquisitions research: A bibliometric study of top strategy and international business journals, 1980-2010. Journal of Business Research, 67(12), 2550-2558. DOI: https://doi.org/10.1016/j.jbusres.2014.03.015

Fu, L., Singhal, R. \& Parkas, M. (2016). Tobin's q Ratio and Firm Performance. International Research Journal of Applied Finance, 7(4). DOI: https://doi.org/10.0704/article-2

Gaughan, P. A. (2009). M\&As in troubling times. Journal of Corporate Accounting \& Finance, 20(2), $45-50$. DOI: https://doi.org/10.1002/jcaf.20465

Ghosh, A. (2004). Increasing market share as a rationale for corporate acquisitions. Journal of Business Finance \& Accounting, 31(1-2), 209-247. DOI: https://doi.org/10.1111/j.0306-686X.2004.0006.x

Gulati, R. (1995). Does familiarity breed trust? The implications of repeated ties for contractual choice in alliances. Academy of Management Journal, 38(1), 85-112. doi: 10.2307/256729. DOI: https://doi.org/10.5465/256729

Haleblian, J., Devers, C., McNamara, G., Carpenter, M., \& Davison, R. (2009). Taking stock of what we know about mergers and acquisitions: A review and research agenda. Journal of Management, 35(3), 469-502. DOI: https://doi.org/10.1177/0149206308330554

Haleblian, J., \& Finkelstein, S. (1999). The influence of organizational acquisition experience on acquisition performance: A behavioral learning perspective. Administrative Science Quarterly, 44(1), 29. DOI: https:// doi.org/ $10.2307 / 2667030$

Haleblian, J., Kim, J., \& Rajagoplan, N. (2006). The influence of acquisition experience and performance on acquisition behavior: Evidence from the u.s. commercial banking industry. Academy of Management Journal, 49(2), 357-370. DOI: https://doi.org/10.2307/20159768

Hannan, T., \& Pilloff, S. (2009). Acquisition targets and motives in the banking industry. Journal of Money, Credit and Banking, 41(6), 1167-1187. DOI: https://doi.org/10.1111/j.1538-4616.2009.00251.x 
Harford, J. (2002). Corporate Cash Reserves and Acquisitions. The Journal of Finance, 54: 1969-1997. DOI: https://doi.org/10.1111/0022-1082.00179

Haunschild, P. R., Davis-Blake, A., \& Fichman, M. (1994). Managerial over-commitment in corporate acquisition processes. Organization Science, 5(4): 528.540.

Hayward, M. (2002). When do firms learn from their acquisition experience? evidence from 1990 to 1995. Strategic Management Journal, 23(1), 21-39. DOI: https://doi.org/10.1002/smj.207

Henningsson, S. (2014). Learning to acquire: how serial acquirers build organisational knowledge for information systems integration. European Journal of Information Systems, 24(2), 121-144. DOI: https://doi.org/10.1057/ ejis. 2014.18

Heron, R., \& Lie, E. 2002. Operating performance and the method of payment in takeovers. Journal of Financial and Quantitative Analysis, 37: 137-155. DOI: https://doi.org/10.2307/3594998

$\mathrm{Hu}, \mathrm{X}$. (2000). Tests for monotonic intensity in a poisson process. Australian \& New Zealand Journal of Statistics, 42(3), 359-365. DOI: https://doi.org/10.1111/1467-842X.00132

Kusewitt, J. B. (1985). An Exploratory Study of Strategic Acquisition Factors Relating to Performance. Strategic Management Journal, 6(2), 151-169.

Levitt, B., \& March, J. (1988). Organizational learning. Annual Review of Sociology, 14(1), 319-338. DOI: https:// doi.org/10.1146/annurev.so.14.080188.001535

Malmendier, U., \& Tate, G. (2008). Who makes acquisitions? CEO overconfidence and the market's reaction. Journal of Financial Economics, 89(1), 20-43. DOI: https://doi.org/10.1016/j.jfineco.2007.07.002

Martynova, M., \& Renneboog, L. (2005). A Century of Corporate Takeovers: What Have We Learned and Where Do We Stand? (previous title: The History of M\&A Activity Around the World: A Survey of Literature). Journal of Banking and Finance, 95. DOI: https://doi.org/10.1016/j.jbankfin.2007.12.038

Masulis, R., Wang, C., \& Xie, F. (2007). Corporate governance and acquirer returns. The Journal of Finance, 62(4), 1851-1889. DOI: https://doi.org/10.1111/j.1540-6261.2007.01259.x

Matsusaka, J. G. (1996) Did Tough Antitrust Enforcement Cause the Diversification of American Corporations? Journal of Financial and Quantitative Analysis, 31, 283-294. DOI: https://doi.org/10.2307/2331183

Nelson, R., \& Winter, S. (1982). An evolutionary theory of economic changeAn evolutionary theory of economic change. Harvard University Press.

Palepu, K. G. (1986). Predicting takeover targets: A methodological and empirical analysis. Journal of Accounting and Economics, 8(1), 3-35. DOI: https://doi.org/10.1016/0165-4101(86)90008-X

Peng, Y., \& Fang, C.-P. (2010). Acquisition experience, board characteristics, and acquisition behavior. Journal of Business Research, 63(5), 502-509. DOI: https://doi.org/10.1016/j.jbusres.2009.04.009

Rhodes-Kropf, M., \& Viswanathan, S. (2004), Market Valuation and Merger Waves. The Journal of Finance, 59(2): 2685-2718.

Rhodes-Kropf, M., \& Robinson, D. T. (2008), The Market for Mergers and the Boundaries of the Firm. The Journal of Finance, 63(3), 1169-1211. DOI: https://doi.org/10.1111/j.1540-6261.2008.01355.x

Roberts, M. R., \& Whited, T. M. (2013). Endogeneity in Empirical Corporate Finance, in George M. Constantinides, Milton Harris \& Rene M. Stulz., eds., Handbook of the Economics of Finance, 493-572.

Rosenbaum, P., \& Rubin, D.B. (1983) The Central Role of the Propensity Score in Observational Studies for Causal Effects. Biometrika, 70(1), 41-55. DOI: https://doi.org/10.1093/biomet/70.1.41

Rossi, S., \& Volpin, P. F. (2004). Cross-country determinants of mergers and acquisitions. Journal of Financial Economics, 74(2), 277-304. DOI: https://doi.org/10.1016/j.jfineco.2003.10.001

Shi, W., Zhang, Y., \& Hoskisson, R. E. (2017). Ripple effects of ceo awards: Investigating the acquisition activities of superstar ceos' competitors. Strategic Management Journal. DOI: https://doi.org/10.1002/smj.2638

Shimizu, K. (2007). Prospect Theory, Behavioral Theory, and the Threat-Rigidity Thesis: combinative effects on organizational decisions to divest formerly acquired units. Academy of Management Journal, 50(6) 14951514. DOI: https://doi.org/10.2307/20159486 
Shleifer, A., \& Visnhy, R. (2003). Stock Market Drives Acquisitions. Journal of Financial Economics, 70(3), 295311. DOI: https://doi.org/10.1016/S0304-405X(03)00211-3

Statista, The Statistics Portal. (2018). Value of mergers and acquisitions (M\&A) worldwide from 2012 to 2017 (in billion U.S. dollars). Disponível em: <http:/www.statista.com/statistics/186337/number-of-mobilebroadband-subscriptions-worldwide-since-2005/>.

Zollo, M., \& Winter, S. (2002). Deliberate learning and the evolution of dynamic capabilities. Organization science, 13(3), 339-351. DOI: https://doi.org/10.1287/orsc.13.3.339.2780

Zollo, M., \& Singh, H. 2004. Deliberate learning in corporate acquisitions: Post-acquisition strategies and integration capability in US bank mergers. Strategic Management Journal, 25: 1233-1256. DOI: https://doi. org/10.1002/smj.426

\section{Como citar este artigo}

Pimenta, D. P., \& Porto, R. B. (2018). Por que as Empresas Realizam Aquisições com Frequência? Revista de Contabilidade e Organizações, 12:e143279. DOI: http://dx.doi.org/10.11606/issn.1982-6486. rco.2018.143279 